\title{
Empirical model for estimating vertical concentration profiles of re-suspended, sediment-associated contaminants
}

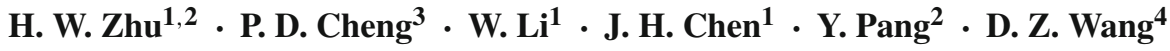

Received: 30 October 2016 / Revised: 30 December 2016 / Accepted: 17 January 2017 / Published online: 7 March 2017

(C) The Chinese Society of Theoretical and Applied Mechanics; Institute of Mechanics, Chinese Academy of Sciences and Springer-Verlag Berlin Heidelberg 2017

\begin{abstract}
Vertical distribution processes of sediment contaminants in water were studied by flume experiments. Experimental results show that settling velocity of sediment particles and turbulence characteristics are the major hydrodynamic factors impacting distribution of pollutants, especially near the bottom where particle diameter is similar in size to vortex structure. Sediment distribution was uniform along the distance, while contaminant distribution slightly lagged behind the sediment. The smaller the initial sediment concentration was, the more time it took to achieve a uniform concentration distribution for suspended sediment. A contaminants transportation equation was established depending on mass conservation equations. Two mathematical estimation models of pollutant distribution in the overlying water considering adsorption and desorption were devised based on vertical distribution of suspended sediment: equilibrium partition model and dynamic micro-diffusion model. The ratio of time scale between the sediment movement and sorption can be used as the index of the models. When this ratio was large, the equilibrium assumption was reasonable, but when it was small, it might require dynamic micro-diffusion model.
\end{abstract}

$\triangle$ P. D. Cheng

pdcheng@imech.ac.cn

1 Shanghai Investigation, Design \& Research Institute Co., Ltd, Shanghai 200434, China

2 Institute of Environmental Science and Engineering, Hohai University, Nanjing 210098, China

3 Key Laboratory for Mechanics in Fluid Solid Coupling System, Institute of Mechanics, Chinese Academy of Science, Beijing 100190, China

4 Shanghai Institute of Applied Mathematics and Mechanics, Shanghai University, Shanghai 200072, China
Keywords Sediment resuspension · Pollutants · Concentration distribution $\cdot$ Desorption $\cdot$ Partition coefficient

\section{Introduction}

Sediments have a very important influence on the enrichment, migration, and transformation of pollutants in the water environment $[1,2]$. On the one hand, sediment particles act as a "sink" of contaminants, on the other hand, they can be a potential source of pollutants [3]. The main components of the suspended sediments are fine-grained particles of large specific surface area, which is the main pollutant transport carrier $[4,5]$. The interaction between sediment and pollutant mainly occurred on two interfaces. One is the surface of sediment particles, which reflects the adsorption and desorption effect $[6,7]$. The other is the sediment-water interface, controlling the material diffusion and exchange between the overlying water and sediments, and also the main place where pollutant transformation occurs in rivers, lakes, and sediments $[8,9]$.

The extent of pollutants in desorption and release rate from sediment will directly lead to the change of pollutant concentration in the water body [10-12]. Meanwhile, the sediment concentration is controlled by the fluid hydrodynamics. The pollutants in sediments are divided into the particle state (solid phase) and water state (liquid phase), between which there is a dynamic equilibrium [13]. This equilibrium state will be broken when sediment particles from the bed surface are washed into the overlying water and mixed with the water flow, which is placed in the water environment with a lowconcentration of pollutants [3]. However, the residence time of particles suspended in the water column is much shorter than that of pollutants desorption. So it is difficult to establish 
the mathematical model of pollutant concentration variation in the overlying water [14]. The main difficulty lies in sediment desorption-adsorption behaviors and are influenced by many factors, such as contaminant characteristics, particle properties, sediment transport, and flow turbulence. As mentioned above, the time scale of desorption is far more than the sediment movement and is very sensitive to the experimental conditions [15]. Vertical pollutants distributions are closely related to suspended sediment. Different sorption capacity of sediment contaminants, particle sizes, and turbulence may cause kinds of spatial distributions. How to get suspended pollutant distribution through suspended sediment distribution is worth studying.

In order to study the migration process of sediment contaminants in water, the single factor method was used to study the vertical distribution process of suspended sediment in the uniform flow. Characteristics of phosphorus adsorption and desorption from the sediment particles based on two estimation models in water were studied by using two different flumes. The circular flume experiments were carried out to meet the specific requirements of the water flow condition and the time scale of desorption.

\section{Materials and methods}

\subsection{Sediment sampling}

Sediments were collected in situ from Dianshan Lake and Suzhou Creek in Shanghai, China (Fig. 1). Particle diameter was measured by a laser particle size analyzer. In these experiments, the organic matter and other impurities need to be removed firstly from the sediment samples containing pollutants, and the specific methods are prescribed in accordance with Ref. [16].

\subsection{Test apparatuses and method}

Suspended sediment movement simulation experiments were conducted in an annular flume and a straight flume. The annular flume can change distance parameters into time parameters to ensure the infinite length of hydraulic conditions for deposition distance requirements of the fine particle sediment, and it will not disturb the flocculation structure and vertical distribution of sediment concentration $[17,18]$. Straight flume provides enough length for sediment settlement along the distance. The annular flume and the straight flume are illustrated in Fig. 2.

The annular flume is made of plexiglass with a diameter of $0.6 \mathrm{~m}$ (inner) and $1.0 \mathrm{~m}$ (outer), resulting in a $0.2 \mathrm{~m}$ wide channel. An annular lid fits inside the channel and touches the water surface, rotating to cause a turbulent flow in the channel. The relationship between flow velocity and shear

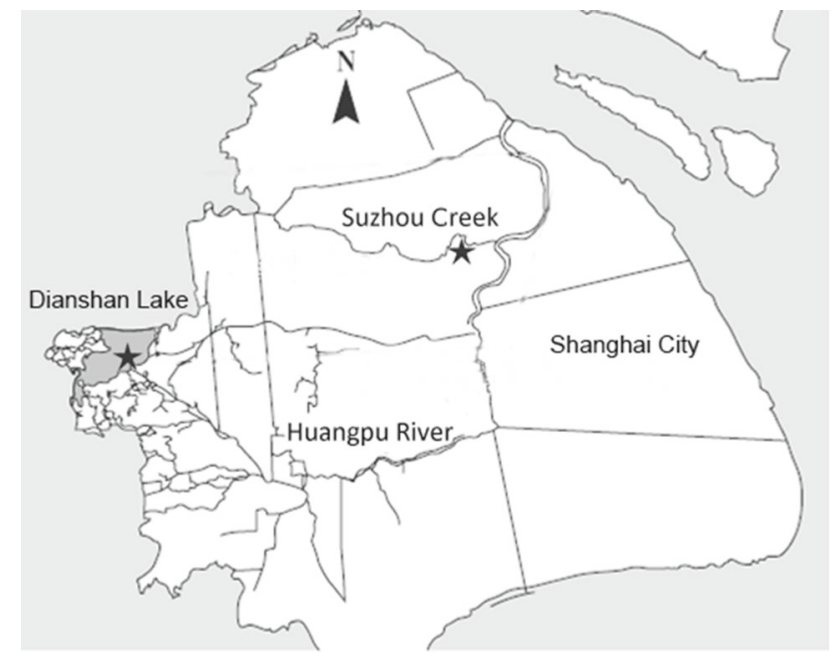

Fig. 1 Map showing sampling site locations

ring speed can be determined by rate calibration. The straight flume is a rectangular test section of $26 \mathrm{~m}$ in length, $0.8 \mathrm{~m}$ in width, and $0.7 \mathrm{~m}$ in height. Water flow was controlled by a variable rotating speed pump and a tail valve. At the beginning of the experiment, sediment samples were set on the bottom of the channel, and the thickness was set as $3-5 \mathrm{~cm}$ to provide sufficient suspended particles. Shear ring speed was adjusted to produce the required velocity until the vertical distribution of sediment concentration was stable. The amount of sediment suspended at a certain velocity was set as initial sediment concentration. Three water sampling ports were arranged in an in-line array. Phosphorus concentration in water was measured by a $722 \mathrm{~N}$ visible spectrophotometer [16]. An intelligent flow meter was used to measure the flow rate. Turbidity meter was used to measure the sediment concentration. The experimental flume conditions and the characteristic values are shown in Table 1.

\section{Theoretical models for suspended sediment transport}

In natural rivers, the molecular diffusivity $D_{m}$ is much smaller than the eddy diffusivity $E(z, t)$, assuming source term $R(c, z, t)=0$, yielding the suspended sediment transport equation

$\frac{\partial c(z, t)}{\partial t}=\omega_{s} \frac{\partial c(z, t)}{\partial z}+\frac{\partial}{\partial z}\left(E(z, t) \frac{\partial c(z, t)}{\partial z}\right)$,

where $c(z, t)$ is the sediment vertical concentration, $\omega_{s}$ is the particle settling velocity, and $D_{e}(z, t)$ is the eddy diffusivity. It can be seen from Eq. (1) that the principal factors affecting the vertical migration and distribution of suspended sediment are the particle settling velocity and the eddy diffusivity. 
a

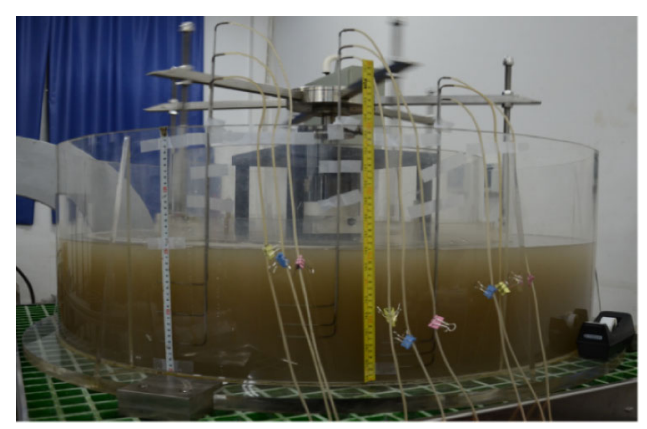

c

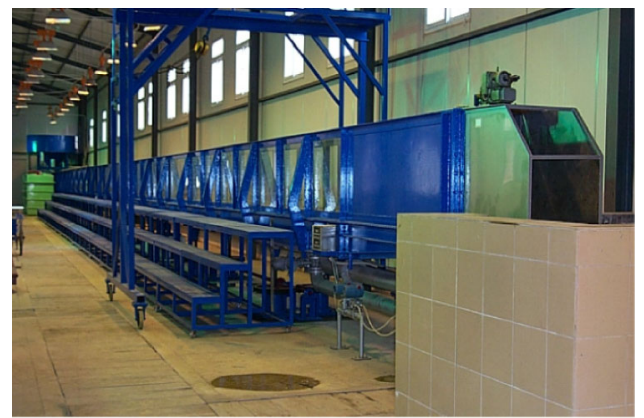

b

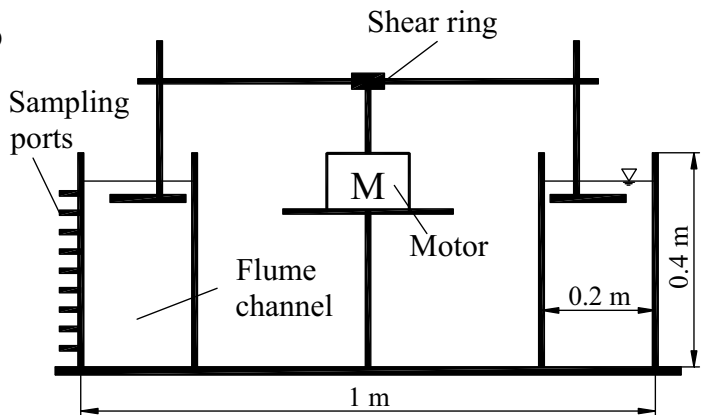

d

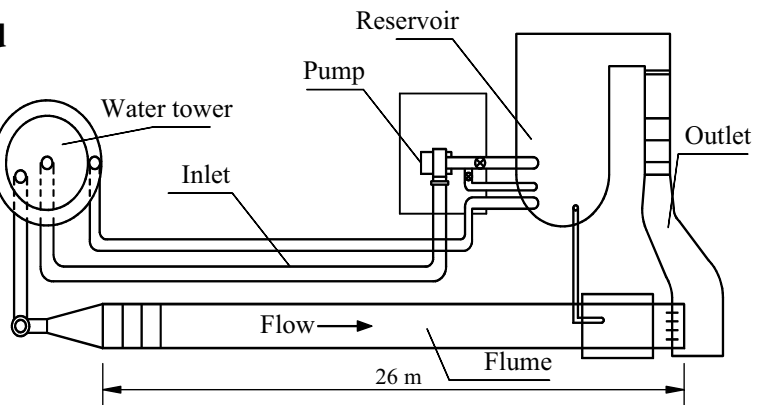

Fig. 2 Schematic locations of the annular flume and the straight flume

Table 1 Velocity, sediment concentration, and pollutant concentration in characterized region

\begin{tabular}{|c|c|c|c|c|c|c|c|c|c|c|}
\hline \multirow{2}{*}{$\begin{array}{l}\text { Flume } \\
\text { type }\end{array}$} & \multirow{2}{*}{$\begin{array}{l}\text { Initial } \\
\text { sediment } \\
\text { content }(\mathrm{g} / \mathrm{L})\end{array}$} & \multirow{2}{*}{$\begin{array}{l}\text { Water } \\
\text { depth (m) }\end{array}$} & \multirow{2}{*}{$\begin{array}{l}\text { Median } \\
\text { diameter } \\
(\mathrm{mm})\end{array}$} & \multirow{2}{*}{$\begin{array}{l}\text { Mean } \\
\text { flow rate } \\
(\mathrm{m} / \mathrm{s})\end{array}$} & \multicolumn{3}{|c|}{ Sediment concentration $(\mathrm{g} / \mathrm{L})$} & \multicolumn{3}{|c|}{ Pollutant concentration $(\mathrm{mg} / \mathrm{L})$} \\
\hline & & & & & $\frac{z}{H}=0.1$ & $\frac{z}{H}=0.5$ & $\frac{z}{H}=0.9$ & $\frac{z}{H}=0.1$ & $\frac{z}{H}=0.5$ & $\frac{z}{H}=0.9$ \\
\hline Annular-1 & 0.5 & 0.2 & 0.02 & 0.15 & 1.563 & 0.781 & 0.391 & 1.515 & 1.507 & 1.441 \\
\hline Annular-2 & 1.5 & 0.2 & 0.02 & 0.15 & 0.586 & 0.391 & 0.195 & 3.580 & 3.442 & 3.169 \\
\hline Annular-3 & 0.5 & 0.2 & 0.03 & 0.15 & - & - & - & 0.21 & 0.15 & 0.13 \\
\hline Straight-1 & 0.5 & 0.5 & 0.1 & 0.43 & 0.1 & 0.05 & 0.02 & - & - & - \\
\hline Straight-2 & 0.5 & 0.5 & 0.025 & 0.48 & 0.1 & 0.07 & 0.06 & - & - & - \\
\hline
\end{tabular}

\subsection{Particle settling velocity}

The particle settling velocity is influenced by its geometry, sediment concentration, and temperature. Assuming that the single particle is spherical with a diameter $d$, its settling velocity can be written as Ref. [19]

$\omega_{s}=\sqrt{\left(c_{1} \frac{v}{d}\right)^{2}+c_{2} \frac{\gamma_{s}-\gamma}{\gamma} g d}-c_{1} \frac{v}{d}$

where $\gamma$ and $\gamma_{s}$ are the specific weight of water and particle, respectively, $v$ is the fluid kinematic viscosity, $d$ is the particle diameter, $c_{1}$ and $c_{2}$ are the empirical coefficients. When $c_{1}=$ 13.95 and $c_{2}=1.09$, Eq. (2) meets the requirements of laminar flow zone, turbulent zone, and transition zone simultaneously. When $d$ is small enough, Eq. (2) can be simplified as $\omega_{s}=1.044 \sqrt{\frac{\gamma_{s}-\gamma}{\gamma} g d}$

Fine-grained particles in the water body tend to be in the form of a flocculation during migration in water. Fractal dimension $D_{f}$ can be used to describe the particle aggregates as follows [20]

$V=C L^{D_{f}}$,

where $V$ is the particle aggregate volume, $C$ is a constant, $L$ is the characteristic length, the maximum length of a particle in general.

Then aggregate effective density $\rho_{f}$ can be represented as [20] 


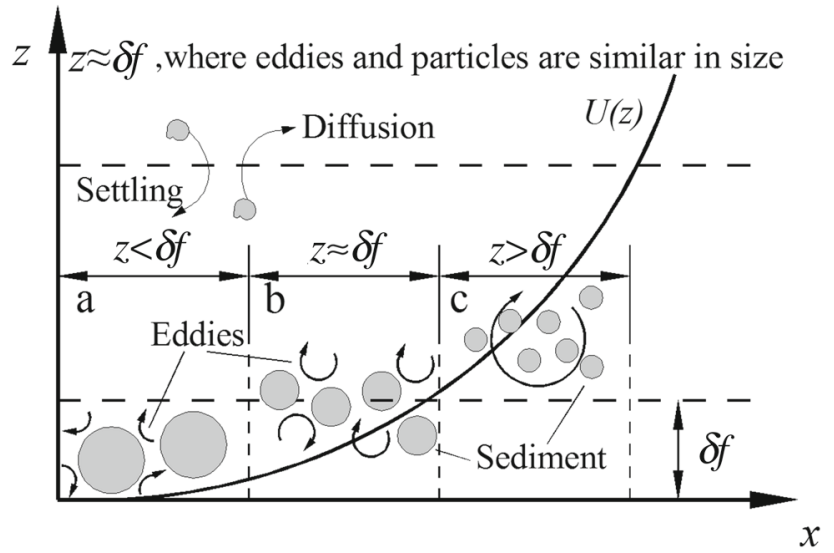

Fig. 3 Comparison of eddies and particles at different location in water

$\rho_{f}-\rho=\left(\rho_{r}-\rho\right)\left(\frac{d_{f}}{d}\right)^{D_{f}-3}$,

where $\rho$ represents the water density, $d_{f}$ is the aggregate diameter.

Substitute Eq. (5) into Eq. (3), and the aggregate settling velocity $\omega_{s f}$ can be written as

$\omega_{s f}=1.044 \omega_{s}\left(\frac{d_{f}}{d}\right)^{D_{f}-1}$,

where $\omega_{s}$ is the settling velocity of single particle. From Eq. (6) we can see that aggregate settling velocity increases with the increasing of the diameter. The fractal dimension in Eq. (4) varies with time and space, which can be neglected in the flow of low turbulence intensity in the practical application. As fine-grained sediments settle in the form of flocculation structure, whose fractal dimension is generally taken as $2-2.5\left(D_{f}=2\right.$ in this paper).

\subsection{Eddy diffusivity}

Turbulent flow will be in the formation of many large and small eddies, the large eddy store energy and transfer energy to the intermediate and small eddy [21]. If the particle size is smaller than this eddy in the turbulence, the particle will remain in suspension [22]. In the turbulence, the particles can be spread in the action of the vortex, and the micor-scale eddy causes shear force between particles. Eddies in the same size of particles make the most effective particle interactions (as shown in Fig. 3) [14].

An eddy that is smaller than the particle size will not take the movement and only affect the pollutant transport condition near the surface of the particles in the surrounding water. In the near bottom region, the eddy diffusion coefficient is related to the surface shear stress and the distance to the bottom surface. When the generation and dissipation of turbulent kinetic energy balance one another locally, then [20]

$E(z, t)= \begin{cases}\kappa u_{*} z, & 0 \leqslant z \leqslant \delta \\ \kappa u_{*} \delta_{b}, & \delta<z \leqslant \delta_{b}\end{cases}$

where $u_{*}$ is the friction velocity, $\delta$ is the height above bottom, $\delta_{b}$ is the beneath boundary layer [20]. $\kappa$ is the Karman constant $(\kappa=0.4)$. When eddies are the same size as particles, $\delta=\delta_{f}=u_{*}^{3} \nu /\left(\kappa \omega_{s f}^{4}\right)$. From Eq. (7), we can see that the eddy diffusion coefficient depends not only on the turbulence, but also the sediment surface roughness and particle movement in the water.

\subsection{Verification of vertical transport equation of suspended sediment}

According to the experimental conditions, particles with diameter less than $0.063 \mathrm{~mm}$ accounted for $80 \%$ of the amount. Settling velocity of the reference particle with diameter $d_{r}=0.063 \mathrm{~mm}$ was calculated by Eq. (3). When sediment particle size is larger than $0.03 \mathrm{~mm}$, flocculation phenomenon may be not observable, thus particle diameter can be considered equal to that of the reference particle $[22,23]$, then flocculation settling velocity can be obtained as $w_{s f}=1.77 \times 10^{-3} \mathrm{~m} / \mathrm{s}$ according to Eq. (6).

From diffusion equation for suspended sediment transportation and the linear distribution of flow shear force in open channel, vertical distribution of sediment reaches an equilibrium state at [14]

$\frac{C}{C_{\delta_{f}}}=\left(\frac{H-z}{z} \frac{\delta_{f}}{H-\delta_{f}}\right)^{w_{s} /\left(\kappa u_{*}\right)}$.

Substitute $\delta_{f}=0.026 \mathrm{~m}$ [14], $C_{\delta_{f}}=0.5 \mathrm{~g} / \mathrm{L}, w_{s f}=1.77 \times$ $10^{-3} \mathrm{~m} / \mathrm{s}, H=0.2 \mathrm{~m}$, and $u_{*}=1.2 \times 10^{-3} \mathrm{~m} / \mathrm{s} \mathrm{into} \mathrm{Eq.} \mathrm{(8)}$ yields

$C(z)=3 \times 10^{-7}\left(\frac{0.2}{z}-1\right)^{7.5}$.

In order to verify the application scope of Eq. (9), theoretical values were compared with the experimental results carried in the long straight flume by substituting related parameters into Eq. (9) as shown in Table 2.

The results arrive at

$C(z)=0.43\left(\frac{0.5}{z}-1\right)^{0.1}$ for $d_{r}=0.025 \mathrm{~mm}$,

and

$C(z)=3 \times 10^{-19}\left(\frac{0.5}{z}-1\right)^{15}$ for $d_{r}=0.1 \mathrm{~mm}$. 
Table 2 Related parameters in Eq. (8) for the experiments carried in long straight flume

\begin{tabular}{lllllll}
\hline & $d_{r}(\mathrm{~mm})$ & $w_{s r}(\mathrm{~m} / \mathrm{s})$ & $w_{s f}(\mathrm{~m} / \mathrm{s})$ & $\delta_{f}(\mathrm{~m})$ & $C_{\delta_{f}}(\mathrm{~g} / \mathrm{L})$ & $H(\mathrm{~m})$ \\
\hline 1 & 0.025 & $0.56 \times 10^{-3}$ & $0.56 \times 10^{-3}$ & 0.086 & 0.5 & 0.5 \\
2 & 0.1 & $8.98 \times 10^{-3}$ & $8.98 \times 10^{-3}$ & 0.001 & 0.5 & 0.015 \\
\hline
\end{tabular}

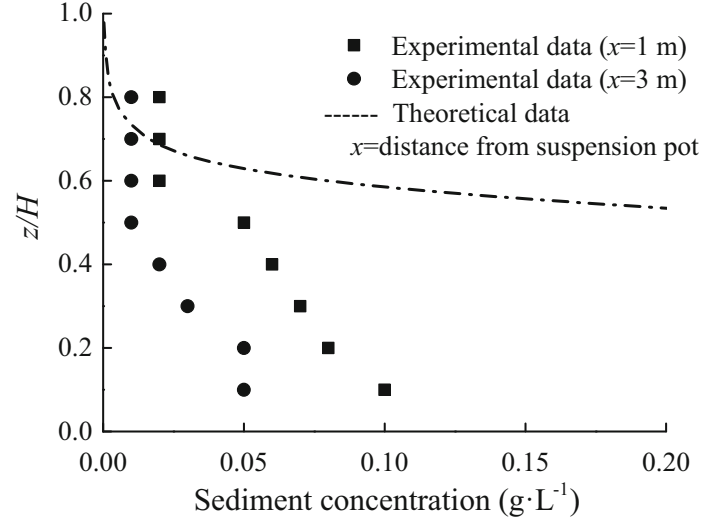

Fig. 4 Comparison of theoretical and experimental results in vertical sediment concentration distribution with sediment particle size of $0.025 \mathrm{~mm}$

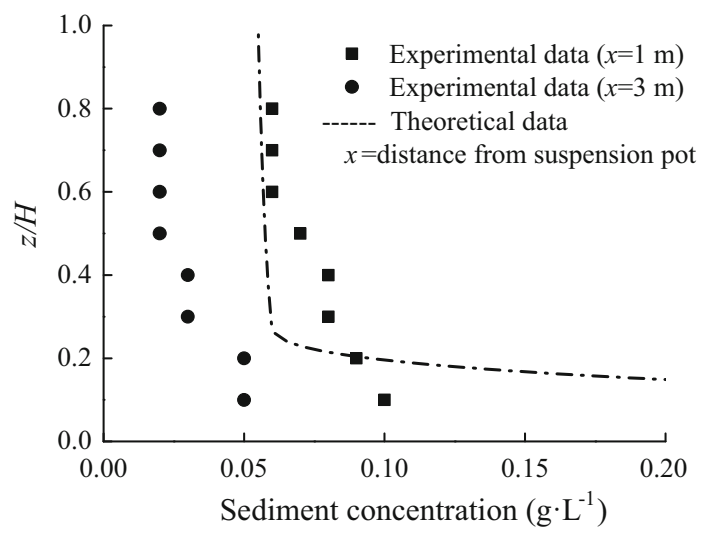

Fig. 5 Comparison of theoretical and experimental results in vertical sediment concentration distribution with sediment particle size of $0.1 \mathrm{~mm}$

The results of Eqs. (10) and (11) are compared with the experimental results in Figs. 4 and 5.

From Figs. 4 and 5 we can see that the sediment concentration near the bottom decayed very fast along the distance. As the particle size decreased, suspended sediment concentration took longer to reach equilibrium during the vertical distribution process. This may be because the particle settling velocity depends on its size, larger particles have higher deposition rate, resulting equilibrium concentration along the shorter horizontal distance. Theoretical values are calculated by using Eq. (9) were not in good accordance with experimental values in the area close to the water surface, especially for particles of $d_{r}=0.1 \mathrm{~mm}$. It may be linked to the drag coefficient of the coarse particles as Eq. (9) derivates in the laminar flow pattern, and the particle settling velocity is relatively large.

\section{Two models for estimating contaminant concentration distribution}

Numerous studies suggest that adsorption process is a complex process, including the fast adsorption and slow adsorption [24-28]. Similarly, desorption is also fast firstly and then tends to be slow. In the "two- step adsorption/desorption model", one of the explanations is that there is a very rapid physical adsorption stage, followed by a slow adsorption and transformation of chemical changes; the other is that the adsorption/desorption occurs first on the surface of the sediment particles, and then in the internal pore structure of the sediment particles [26]. In this paper, the second explanation was used to build the adsorption and desorption model.

Total contaminant concentration $C_{t}(\mathrm{mg} / \mathrm{L})$ is defined as the sum of the particulate $C_{p}(\mathrm{mg} / \mathrm{L})$ and the dissolved phase $C_{d}(\mathrm{mg} / \mathrm{L})$ concentrations [14],

$C_{t}=C_{p}+C_{d}$.

As $C_{m}$ is set to the sediment concentration (mass sediment per unit volume of water, $(\mathrm{g} / \mathrm{L})), C_{S}$ is the concentration in the solid phase (mass compound per unit of solid, (mg/g)), then $C_{p}=C_{m} C_{s}$, the partition coefficient $K_{p}$ can be defined as

$K_{p}=\frac{C_{s}}{C_{d}}=\frac{C_{p}}{C_{m} C_{d}}$.

At present, the mathematical description of the "two-step adsorption and desorption model" can be used in two ways. The first is the equilibrium partition model, and the second is the dynamic micro-diffusion model, in which sediment sorption is considered a "diffusion" behavior. Then the above two models are used to predict the concentration distribution of pollutants in the overlying water as follows.

\subsection{Equilibrium partition model}

As mentioned above, the two-phase partition process of pollutants in overlying water is a fast-easy step and a slow-hard one. If adsorption/desorption equilibrium is reached at once when the sediment pollutant is suspended and enters the 
Table 3 Fitting parameters of the Langmuir model for desorption equilibrium experiments

\begin{tabular}{|c|c|c|c|c|c|c|}
\hline \multirow[t]{2}{*}{ No. } & \multirow{2}{*}{$\begin{array}{l}\text { Sediment } \\
\text { concentration } \\
\left(\mathrm{kg} / \mathrm{m}^{3}\right)\end{array}$} & \multicolumn{2}{|c|}{ Langmuir model parameters } & \multirow{2}{*}{$\begin{array}{l}\text { Adsorption } \\
\text { rate } k_{1} \\
(\mathrm{mg} \mathrm{L} / \mathrm{s})\end{array}$} & \multirow{2}{*}{$\begin{array}{l}\text { Desorption } \\
\text { rate } k_{2} \\
(\mathrm{mgL} / \mathrm{s})\end{array}$} & \multirow{2}{*}{$\begin{array}{l}\text { Partition } \\
\text { coefficient } \\
K_{p}(\mathrm{~L} / \mathrm{mg})\end{array}$} \\
\hline & & $b(\mathrm{mg} / \mathrm{g})$ & $k(\mathrm{mg} / \mathrm{L})$ & & & \\
\hline 1 & 0.5 & 2.722 & 0.444 & 0.374 & 0.166 & 4.506 \\
\hline 2 & 1 & 1.021 & 0.236 & 1.113 & 0.261 & 4.264 \\
\hline
\end{tabular}

water without considering the sediment inner pore structure, the simplest way to describe the adsorption rate and the desorption rate is a first-order reaction.

The adsorption and desorption processes were fitted by Langmuir adsorption isotherm equation (1) and Langmuir adsorption kinetic equation (2), as shown below [27,28]

$N_{\infty}=b \frac{C_{\infty}}{k+C_{\infty}}$,

$\frac{\partial N}{\partial t}=k_{1}(b-N)-k_{2} N$,

where $N$ and $N_{\infty}$ are the equilibrium adsorption capacities per unit of solid sediment, $C_{\infty}$ is the equilibrium concentration in water phase, $b$ is the saturated adsorption capacity, $k$ is the rate constant of adsorption/desorption, $k_{1}$ and $k_{2}$ are respectively the adsorption rate and the desorption rate.

Because of the linear relationship between the adsorption flux and $k_{1} C_{d}$, also for the desorption flux and $k_{2} C_{p}$, the adsorption flux is equal to the desorption flux as $k_{2} C_{p} \approx$ $k_{1} C_{d}$, so

$C_{m} K_{p}=\frac{k_{1}}{k_{2}}$.

The experimental results of the desorption equilibrium of fine particles are shown in Table 3 [3].

When sediment particles are suspended, the adsorption and desorption equilibrium processes between original sediment and pore water are broken, dissolved contaminants are first released from sediment into the overlying water. Rebalancing process of adsorption and desorption occurs between the particle surface and the water body, contaminants come from the released pore water and desorption from sediment particles. Assuming that suspended sediment concentration is saturated, the amount of dissolved pollutants in the water body is described as

$C_{w}=\frac{C_{0} b}{H K_{p}}+\frac{C(z) b}{K_{p}}$.

Substitute the initial sediment concentration $C_{0}=0.5 \mathrm{~g} / \mathrm{L}$, the partition coefficient $K_{p}=4.506 \mathrm{~L} / \mathrm{mg}$, water depth $H=$ $0.2 \mathrm{~m}$, the saturated adsorption capacity $b=2.722 \mathrm{mg} / \mathrm{g}$ and Eq. (11) into Eq. (17), the result was shown in Fig. 6.

The results in Fig. 6 show that the vertical distribution of pollutant concentrations in the initial time could be well

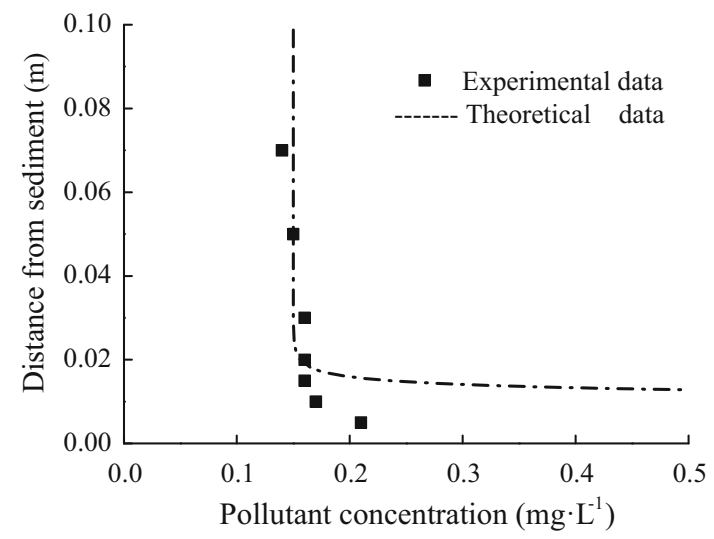

Fig. 6 Comparison of suspended sediment concentration from experiments and partition model (straight flume-1)

fitted by the equilibrium partition model, but the pollutant concentration near the bottom was too far from the estimated model. There might be two reasons for this. The first is that the "two-step adsorption and desorption model" is an initial fast desorption process, and is well fitted by the model. Second, when the sediment concentration near the bottom is too high, the adsorption and desorption processes between suspended particles are influenced by each other. Moreover, some of the particles were in adsorption non-equilibrium state, which first adsorbs pollutants, resulting in low pollutant concentration at the sediment-water interface.

Combining Eqs. (14) and (15), the change of pollutant concentration in the overlying water is obtained and illustrated in Fig. 7. From the results, it can be seen that the vertical distribution of pollutants near the bottom has a distinct concentration hierarchical structure, where large deviation exists between the experimental and theoretical values. With increasing time, the theoretical value is still less than the experimental one. This may be due to the increase of time, uniform distribution of vertical sediment, and increased potential sources in the upper layer. The difference between theoretical and experimental values is smaller with time, which indicates that although the adsorption and desorption of sediment particles are complex, the theoretical derivation can still describe the overall pollutants distribution process in the overlying water. 


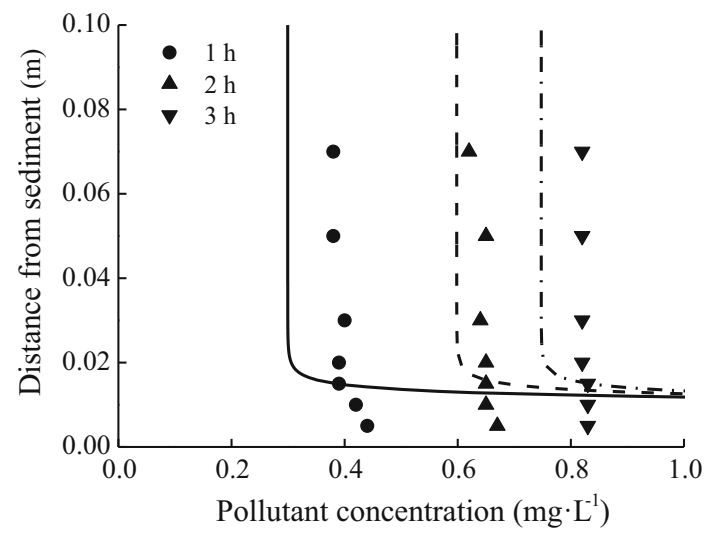

Fig. 7 Comparison of suspended sediment concentration variation from experiments and equilibrium partition model (straight flume-1)

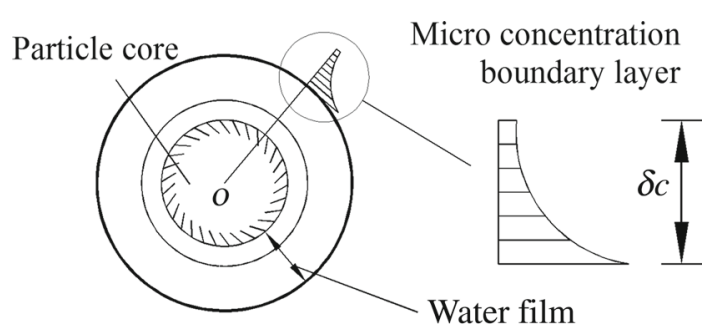

Fig. 8 Microcosm in sediment particle surface

\subsection{Dynamic micro-diffusion model}

The parameters in Eq. (17) for describing the desorption kinetics have to be determined by experiments. An effective diffusion parameter can be used to refer the adsorption and desorption process based on the mathematical model, which is a function of molecular diffusion coefficient, distribution coefficient, pore structure, and particle density [22]. The model suggests that the pollutant desorption process occurs in microscopic particles and pore water, as well as between the particles and the macro water, as shown in Fig. 8

For a spherical particle of radius $R$, the mathematical expression of these transport processes can be described as [14]

$$
\begin{gathered}
\frac{\partial}{\partial t}\left\{\left[(1-n) \rho_{s} K_{p}+n\right] C_{1}(r, t)\right\} \\
=\frac{1}{r^{2}} \frac{\partial}{\partial r}\left(r^{2} D_{e 1} T \frac{\partial C_{1}(r, t)}{\partial r}\right),
\end{gathered}
$$

where $C(r, t)$ is the local volumetric water phase concentration, $n$ is the porosity, $\rho_{s}$ is the mass density of the solid particles, $r$ is the radial distance, Eq. (18) is valid for $0<r<R$.

When turbulence is low intensity, an exterior boundary layer of thickness $L_{D}$ limits of mass exchange between the water and the suspended sediment. Transport process through this layer is governed by

$\frac{\partial C_{2}(r, t)}{\partial t}=\frac{1}{r^{2}} \frac{\partial}{\partial r}\left(r^{2} D_{e 2} \frac{\partial C_{2}(r, t)}{\partial r}\right)$.

Equation (19) is valid for $R<r<R+L_{D}$.

Contaminant transport from inner particles to a water body undergoes two different processes. At the particle-water interface, mass conservation requires that there is no accumulation of the diffusing substance, which can be stated as

$\left.C_{1}(r, t)\right|_{r=R}=\left.C_{2}(r, t)\right|_{r=R}$,

$\left.n D_{e 1} \frac{\partial C_{1}(r, t)}{\partial r}\right|_{r=R}=\left.D_{e 2} \frac{\partial C_{2}(r, t)}{\partial r}\right|_{r=R}$.

Because the diffusion time scale is far larger than the sediment transport, the entire diffusion process can be regarded as a stable state. In reality, when a lot of contaminated sediment is entrained in the water, people consented to what extent the contaminant is desorbed while the sediments are in suspension ignoring how the contaminant releasing into the water. Boundary conditions associated with Eqs. (18) and (19) include zero flux at the center of the particle and the diffusion layer is

$\frac{\partial C}{\partial t}(r, t)=0, \quad C(R, t)=C_{a}, \quad C\left(R+L_{D}, t\right)=C_{w}$,

where $C_{a}$ is the contaminant concentration in particle surfaces, $C_{w}$ is the contaminant concentration in surrounding water phase.

As to the stable diffusion, Eq. (18) can be expressed as

$r^{2} D_{e 2} \frac{\partial C_{2}(r, t)}{\partial r}=0$

Substituting the boundary conditions into Eq. (22), yields

$$
\begin{aligned}
C_{2}(r, t)= & -\frac{R\left(R+L_{D}\right)}{r L_{D}}\left(C_{w}-C_{a}\right) \\
& +\frac{\left(R+L_{D}\right) C_{w}-R C_{a}}{L_{D}} .
\end{aligned}
$$

The transport flux $J_{i}$ for a single particle in unit time can be expressed as

$J_{i}=-D_{e 2} \frac{\mathrm{d} C}{\mathrm{~d} r} \cdot 4 \pi r^{2}=-4 \pi D_{e 2} R\left(R+L_{D}\right) \frac{C_{w}-C_{a}}{L_{D}}$.

As mentioned above, when the particle size is equal to the size of the turbulent vortex, the vortex affects the interaction between the particles, and then 


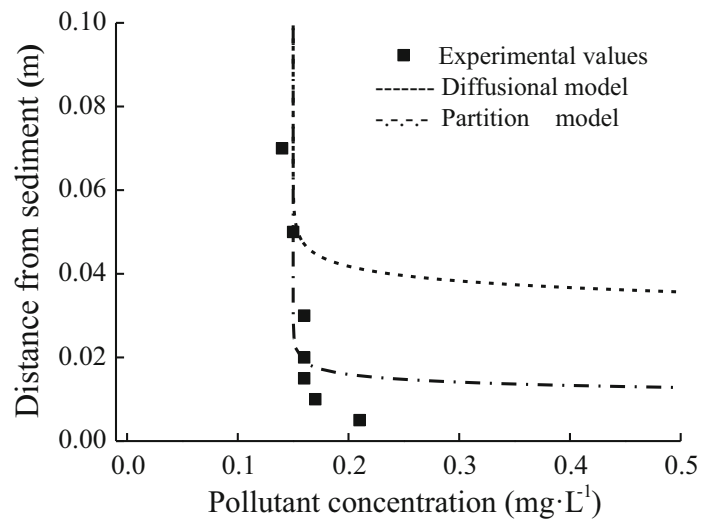

Fig. 9 Comparison of diffusion mode, distribution mode, and experimental value

$L_{D}=L_{k}=2 R$.

Substituting Eq. (25) into Eq. (24), and rearrangement yields

$J_{i}=-6 \pi D_{e 2} R\left(C_{w}-C_{a}\right)$.

Replacing the effective diffusion coefficient with the molecular diffusion coefficient, particle aggression diameter $R$ is $d_{s f} / 2$, overlying water pollutant concentration $C_{w}=0$, and dissolved contaminants concentration $C_{a}=$ $C_{d}$. Substituting the above equations into Eq. (26), pollutants concentration distribution can be defined as

$J(z)=\sum_{i=1}^{m} J_{i}(z)=-\frac{C(z)}{(1-n) \rho_{s}+n \rho} \frac{9}{8 d_{s f}^{2}} D_{m} \frac{b}{K_{P}}$.

Pollutant vertical distribution in the overlying water was predicted by using Eq. (27), as shown in Fig. 9. From the results, it can be seen that the diffusion model fitted well with experimental results, and the diffusion model has a large deviation between theoretical and experimental values. This may be because the stable diffusion model ignored the time for the sorption exchange.

As mentioned above, the main concern is to what extent the contaminant desorbed to the water. Equilibrium assumption may result in the wrong estimation of sediment release. As the source of the sediment and pollutant transport in the water environment system, the distribution of pollutants in the water is of great importance [14].

An average resuspension time $t_{r}$ can be estimated simply as the particle raised height $\delta_{f}$ above the bottom, divided by settling velocity as

$t_{r}=\frac{\delta_{f}}{\omega_{s}}$.

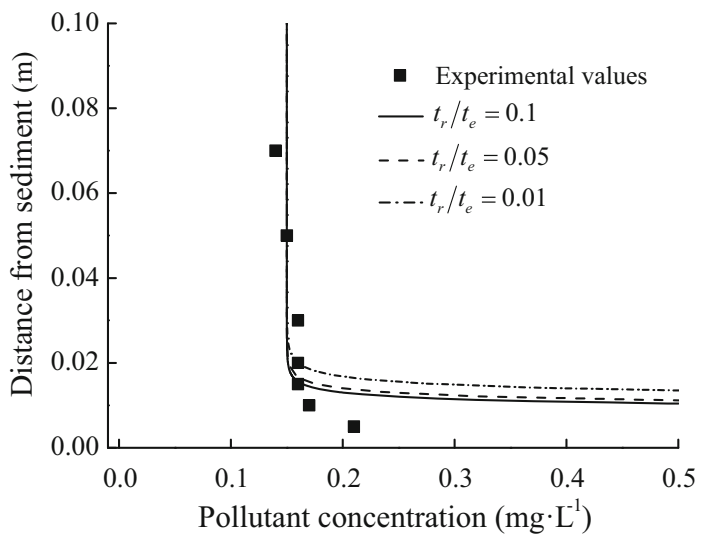

Fig. 10 A modified diffusion model considering suspended sediment time

Suspended sediment particles undergo kinetic desorption at a rate given by the inverse of a time scale to reach equilibrium partitioning, $t_{e}$ (couple of hours for phosphorus). The ratio $\left(t_{r} / t_{e}\right)$ indicates the degree to which equilibrium partitioning assumption would be used. When this ratio is large, the equilibrium assumption is reasonable, but when it is small, the dynamic diffusion model needs to be considered. Substituting $t_{r} / t_{e}$ as a parameter into Eq. (28), the result is shown in Fig. 10. We see that with the increase of $t_{r} / t_{e}$, diffusion model values are closer to the experimental values.

\section{Conclusion}

Many factors can influence contaminant transportation in sediment; therefore, it is difficult to predict the distribution of pollutant concentration in the overlying water. Settling velocity and turbulent disturbance characteristics are the two main factors affecting the vertical distribution of the suspended sediment. Pollutants and suspended sediment have a similar trend in the vertical distribution process, and the vertical concentration of the suspended particles gradually becomes smaller while phosphorus concentration is basically synchronous. Two mathematical models of the release flux established according to the adsorption and desorption effects between pollutants and suspended sediment. The equilibrium partition model is more intuitive considering the characteristics of the pollutant itself, but there is a lack of description for the specific physical processes. The dynamic micro-diffusion model fits well with the diffusion process, but the prediction value of the particles is too small due to neglecting of the diffusion process in the particles and sizeable vortex influence on the particles.

Acknowledgements The project was supported by the National Natural Science Foundation of China (Grant 11502138), the National Key Research and Development Program (Grant 2016YFC0401500), and 
the National Water Pollution Control and Treatment Science and Technology Major Project (Grant 2012ZX07506-002b).

\section{References}

1. Qin, B.Q.: Approaches to mechanisms and control of eutrophication of shallow lakes in the middle and lower reaches of the Yangze River. J. Lake Sci. 03, 193-202 (2002)

2. Cheng, P.D., Zhu, H.W., Zhong, B.C., et al.: Transport mechanisms of contaminants released from fine sediment in rivers. Acta Mech. Sin. 31, 791-798 (2015)

3. Zhu, H.W., Jiang, J.A., Cheng, P.D., et al.: Mechanism of pollutant release due to sediment re-suspension. Adv. Water Sci. 24, 537-542 (2013)

4. Fang, H.W., Huang, L., Wang, J.Y., et al.: Environmental assessment of heavy metal transport and transformation in the Hangzhou Bay, China. J. Hazard. Mater. 302, 447-457 (2016)

5. Huang, L., Fang, H., Reible, D.: Mathematical model for interactions and transport of phosphorus and sediment in the Three Gorges Reservoir. Water Res. 85, 393-403 (2015)

6. Li, B., Zhang, K., Zhong, B.C., et al.: An experimental study on release of pollutants from sediment under hydrodynamic conditions. Chin. J. Hydrodyn. 23, 126-133 (2008)

7. Zhu, G.W., Gao, G., Qin, B.Q.: Geochemical characteristic of phosphorus in sediment of a large shallow lake. Adv. Water Sci. 14, 714-719 (2003)

8. Liu, J., Zheng, X.L., Chen, L., et al.: Study on flux and release law of nitrogen and phosphorus of sediment in reservoir. J. Hydraul. 43, 339-343 (2012)

9. Chatelain, M., Guizien, K.: Modeling coupled turbulencedissolved oxygen dynamics near the sediment-water interface under wind waves and sea swell. Water Res. 44, 1361-1372 (2010)

10. Liang, W., Wang, Z., Jiao, Z.X., et al.: Adsorption of phosphorus in sediment re-suspension under sudden expansion flow conditions. J. Hydrodyn. 25, 112-117 (2013)

11. Shi, Z., Ling, H.L.: Vertical profiles of fine suspension concentration in the Changjiang esturary. J. Sediment. Res. 2, 59-64 (1999)

12. Wang, J.S., Chen, L., Liu, L., et al.: Experimental study of feature of the cohesive sediment lamination movement. Adv. Water Sci. 19, 13-18 (2008)

13. Zhu, H.W., Cheng, P.D., Wang, D.Z., et al.: Hydrodynamic effects on contaminants release due to rususpension and diffusion from sediments. J. Hydrodyn. 25, 731-736 (2013)
14. Rubin, H., Atkinson, J.: Environmental Fluid Mechanics. Marcel Dekker, New York (2001)

15. Zhu, H.W., Cheng, P.D., Zhong, B.C., et al.: The mechanisms of contaminants release due to incipient motion at sediment-water interface. Sci. China Phys. Mech. Astron. 57, 1563-1568 (2014)

16. Zhang, K., Cheng, P.D., Zhong, B.C., et al.: Total phosphorus release from bottom sediments in flowing water. J. Hydrodyn. 24, 589-594 (2012)

17. Zhu, H.W., Cheng, P.D., Wang, D.Z.: Dynamic release process of pollutants during suspended sediment transport in aquatic system. Appl. Math. Mech. 35, 1411-1420 (2014)

18. Huang, J.Z., Ge, X.P., Yang, X.F., et al.: Remobilization of heavy metals during the resuspension of Liangshui River sediments using an annular flume. Chin. Sci. Bull. 57, 2015-2021 (2012)

19. Wilbert, L.: Sediment and Contaminant Transport in Surface Waters. Taylor and Francis Group, New York (2009)

20. Duren, L.A., Middelburg, J.J.: The Benthic boundary layer: transport processes and biogeochemistry. Eos Trans. Am. Geophys. Union 82, 658-659 (2001)

21. Lei, X.L., Han, Y.X., Ran, B., et al.: Impact of environmental factors on contaminants release from the sediment of three gorges reservoir area. Environ. Eng. 01, 198-206 (2016)

22. Bainbridge, Z.T., Lewis, S.E., Smithers, S.G., et al.: Finesuspended sediment and water budgets for a large, seasonally dry tropical catchment: Burdekin River catchment, Queensland, Australia. Water Resour. Res. 21, 9067-9087 (2014)

23. Wang, C., Wang, C.: Turbulent characteristics in open-channel flow with emergent and submerged macrophytes. Adv. Water Sci. 06, 816-822 (2010)

24. Maggi, F., Tang, F.H.M.: Analysis of the effect of organic matter content on the architecture and sinking of sediment aggregates. Mar. Geol. 363, 102-111 (2015)

25. Hu, Y., Kuhn, N.J.: Erosion-induced exposure of SOC to mineralization in aggregated sediment. Catena 24, 517-525 (2016)

26. Fang, H.W., Chen, M.H., Chen, Z.H.: Surface Characteristics and Model of the Environment Sediment. Science Press, Beijing (2009)

27. Banerjee, S., Kumar, A., Maiti, S.K., et al.: Seasonal variation in heavy metal contaminations in water and sediments of Jamshedpur stretch of Subarnarekha river, India. Environ. Earth Sci. 75, 1-12 (2016)

28. Kocan, A.A., Shaw, M.G., Edwards, W.C., et al.: Heavy metal concentrations in the kidneys of white-tailed deer in Oklahoma. J. Wildl. Dis. 16, 593-596 (2015) 